\title{
Author index (Volume 14)
}

Bianchini, R. \& Natalini, R., Well-posedness of a model of nonhomogeneous compressibleincompressible fluids

Dias, J.-P. \& Oliveira, F., On a quasilinear nonlocal Benney system

Fanelli, F., Some local questions for hyperbolic systems with non-regular time dependent coefficients

Fang, Y.-F., Shih, H.-W. \& Wang, K.-H., Local well-posedness for the quantum Zakharov system in one spatial dimension

Folino, R., Slow motion for a hyperbolic variation of Allen-Cahn equation in one space dimension

Frid, H., An extension of Bakhvalov's theorem for systems of conservation laws with damping

Garavello, M. \& Villa, S., The Cauchy problem for the Aw-Rascle-Zhang traffic model with locally constrained flow

$\mathrm{Hu}, \mathrm{Y} .$, Global solutions to a nonlinear wave system arising from cholesteric liquid crystals

Huh, H., Conformal identity of the ChernSimons gauged wave equations and its applications

Jamróz, G., On measures of accretion and dissipation for solutions of the

Camassa-Holm equation

4 (2017) 721

Karlsen, K. H. \& Towers, J. D., Convergence of a

3 (2017) 487

1 (2017) 135

2 (2017) 301

1 (2017) 157

1 (2017) 1

4 (2017) 703

3 (2017) 393

1 (2017) 27

2 (2017) 341
Godunov scheme for conservation laws with a discontinuous flux lacking the crossing condition

Koley, U., Risebro, N. H., Schwab, C. \& Weber, F., A multilevel Monte Carlo finite difference method for random scalar degenerate convection-

Lai, G., Interaction of

Lisibach, A., Characteristic

Ma, Y., Global solutions of

Ma, Y., Global solutions of

Mohammed, M., diffusion equations fan-jump-fan composite waves in a two-dimensional steady jet for van der Waals gases initial value problem for spherically symmetric barotropic flow quasilinear wave-KleinGordon system in twospace dimension:

Technical tools quasilinear wave-KleinGordon system in twospace dimension: Completion of the proof Homogenization of nonlinear hyperbolic stochastic equation via Tartar's method 2 (2017) 323

3 (2017) 415

1 (2017) 73

4 (2017) 565

4 (2017) 591

4 (2017) 627 Trebeschi, P., Existence of approximate current-vortex sheets 
near the onset of instability

Morando, A., Secchi, P. \&

Trebeschi, P., Data dependence of approximate current-vortex sheets near the onset of instability

Natalini, R., see Bianchini, $\mathrm{R}$.

Oliveira, F., see Dias, J.-P.

Risebro, N. H., see Koley, $\mathrm{U}$.

Roy, T., A weak form of the soliton resolution conjecture for highdimensional fourthorder Schrödinger equations

Saalmann, A., Asymptotic stability of $N$-solitons in the cubic NLS equation

Santos, M. M., Reduction of generalized Young measures
3 (2017) 517

3 (2017) 487

1 (2017) 135

2 (2017) 193

3 (2017) 415

2 (2017) 249

3 (2017) 455

2 (2017) 349
Schwab, C., see Koley, U.

Secchi, P., see Morando, A.

Secchi, P., see Morando, A.

Shih, H.-W., see Fang,

$$
\text { Y.-F. }
$$

Towers, J. D., see Karlsen, K. H.

Trebeschi, P., see Morando, A.

Trebeschi, P., see Morando, A.

Villa, S., see Garavello, M.

Wang, K.-H., see Fang, Y.-F.

Weber, F., see Koley, U.

Wei, C., Classical solutions to the relativistic Euler equations for a linearly degenerate equation of state

Zeng, Y., Global existence theory for general hyperbolic-parabolic balance laws with application
3 (2017) 415

2 (2017) 193

3 (2017) 517

1 (2017) 157

4 (2017) 671

2 (2017) 193

3 (2017) 517

3 (2017) 393

1 (2017) 157

3 (2017) 415

3 (2017) 535

2 (2017) 359 